\title{
ON $H^{p}\left(\mathbf{R}^{n}\right)$-MULTIPLIERS OF MIXED-NORM TYPE
}

\author{
C. W. ONNEWEER AND T. S. QUEK
}

(Communicated by J. Marshall Ash)

\begin{abstract}
For a function $m$ in $L^{\infty}\left(\mathbf{R}^{n}\right)$, an appropriately chosen function $\eta$ in $C^{\infty}\left(\mathbf{R}^{n}\right)$ and $\delta>0$ we define $m_{\delta}$ by $m_{\delta}(\xi)=m(\delta \xi) \eta(\xi)$. We show that if $0<p \leq 1$ and if the sequence $\left(\left(m_{2^{n}}\right)^{\wedge}\right)$ belongs to a certain mixed-norm space, depending on $p$, then $m$ is a Fourier multiplier for the corresponding Hardy space $H^{p}\left(\mathbf{R}^{n}\right)$. Moreover, we prove the sharpness of our multiplier theorem. Comparable results had been proved earlier for multipliers for Hardy spaces defined on a locally compact Vilenkin group.
\end{abstract}

\section{INTRODUCTION}

In their main theorem for multipliers for Hardy spaces defined on $\mathbf{R}^{n}$, Baernstein and Sawyer [1] expressed the restrictions imposed on the multipliers of $H^{p}\left(\mathbf{R}^{n}\right), 0<p<1$, in terms of the "norms" of the Herz spaces $K_{1}^{n(1 / p-1), p}$. More specifically, given $m \in L^{\infty}\left(\mathbf{R}^{n}\right)$ and $\eta \in C^{\infty}\left(\mathbf{R}^{n}\right)$ so that $\operatorname{supp}(\eta) \subset\{1 / 4 \leq|\xi| \leq 4\}, 0 \leq \eta(\xi) \leq 1$ for all $\xi \in \mathbf{R}^{n}$, and $\eta(\xi)=1$ on $\{1 / 2 \leq|\xi| \leq 2\}$, let $m_{\delta}(\xi):=m(\delta \xi) \eta(\xi)$ for $\delta>0$. Then Baernstein and Sawyer proved the following.

Theorem B \& S. Let $0<p<1$. Let $m \in L^{\infty}\left(\mathbf{R}^{n}\right)$ and assume that $\sup _{\delta>0}\left\|\left(m_{\delta}\right)^{\wedge}\right\|_{K_{1}^{n(1 / p-1), p}}<\infty$. Then $m \in \mathscr{M}\left(H^{p}\right)$.

This result does not extend to $p=1$, that is to say, there exists an $m \in L^{\infty}$ such that $\sup _{\delta>0}\left\|\left(m_{\delta}\right)^{-}\right\|_{1}<\infty$ and $m \notin \mathscr{M}\left(H^{1}\right)$. Discretizing this statement it becomes: there exists an $m \in L^{\infty}$ such that $\left(\left(m_{2^{j}}\right)^{\wedge}\right)_{j=-\infty}^{\infty} \in l^{\infty}\left(L^{1}\right)$ and $m \notin \mathscr{M}\left(H^{1}\right)$.

The notation used here is: if $0<p, q \leq \infty$ and if $\left(f_{k}\right)_{k=-\infty}^{\infty}$ is a sequence of complex-valued Lebesgue measurable functions on $\mathbf{R}^{n}$ then $\left(f_{k}\right) \in l^{q}\left(L^{p}\right)$, a so-called mixed-norm space, if

$$
\left\|f_{k}\right\|_{l q\left(L^{p}\right)}:=\left(\sum_{k=-\infty}^{\infty}\left\|f_{k}\right\|_{p}^{q}\right)^{1 / q}<\infty,
$$

with the usual modification if $q=\infty$.

Received by the editors September 29, 1992.

1991 Mathematics Subject Classification. Primary 42B15, 42B30.

Key words and phrases. Hardy spaces, Fourier multipliers, mixed norms. 
To deal with the case $p=1$ Baernstein and Sawyer modified the definition of the Herz spaces and introduced spaces $K(w)$. Using the $K(w)$-space norms instead of the $K$-space norms they proved a result similar to Theorem B \& S for $H^{1}$-multipliers, see [1] for further details.

In [3] the present authors proved an analogue of Theorem B \& $\mathbf{S}$ for multipliers on the Hardy spaces $H^{p}(G), 0<p<1$, where $G$ is a locally compact Vilenkin group. In fact, in this setting we obtained a somewhat sharper result than Theorem B \& S, because we were able to replace the $K_{1}^{n(1 / p-1), p}$-norms by $K(1 / p-1 / r, r, p ; G)$-norms for any $r>p$. To deal with the case $p=1$, instead of working with modified $K$-spaces, we considered mixed-norm spaces that were more restrictive than the $l^{\infty}\left(L^{1}\right)$ spaces. Doing this we obtained a multiplier theorem in which the restriction on the multiplier was expressed in terms of certain mixed norms. Moreover, the resulting multiplier theorem applied to all values of $p$ with $0<p \leq 1$; see [3, Corollary 2.3]. These results raised the question of whether it was possible to prove comparable results for $H^{p}\left(\mathbf{R}^{n}\right)$-multipliers, $0<p \leq 1$. In this paper we present our answer to this question.

\section{2. $H^{p}\left(\mathbf{R}^{n}\right)$-MULTIPLIERS, $0<p \leq 1$}

We begin this section with a brief review of the notation we shall use; much of it is the same as the notation used in [1].

For each $j \in \mathbf{Z}$, let

$$
A_{j}=\left\{x \in \mathbf{R}^{n}: 2^{j} \leq|x|<2^{j+1}\right\}
$$

and let

$$
\bar{A}_{j}=A_{j-1} \cup A_{j}=\left\{2^{j-1} \leq|x|<2^{j+1}\right\} .
$$

For a set $E, \chi_{E}$ will denote its characteristic function, and if $E \subset \mathbf{R}^{n}$ is Lebesgue measurable then $|E|$ will represent its Lebesgue measure. As usual $C$ will denote a constant that may change in value from one occurrence to the next. The Fourier transform of a tempered distribution $f$, i.e., $f \in \mathscr{S}^{\prime}$, is denoted by $\hat{f}$; if $f \in L^{1}\left(\mathbf{R}^{n}\right)$ then $\hat{f}(\xi)=\int f(x) e^{-2 \pi i x \cdot \xi} d x$. The inverse Fourier transform is denoted by $\vee$.

A function $m \in L^{\infty}\left(\mathbf{R}^{n}\right)$ is a (Fourier) multiplier for $H^{p}\left(\mathbf{R}^{n}\right), 0<p \leq 1$, if the mapping $f \rightarrow(m \hat{f})^{\vee}$, initially defined on $L^{2} \cap H^{p}$, maps $H^{p}$ continuously into $H^{p}$; we use the notation $m \in \mathscr{M}\left(H^{p}\right)$.

Since our proof of Theorem 2.2 uses the atomic decomposition property of the $H^{p}$ spaces, we give here the definition of a $(p, \infty)$ atom. A function $a: \mathbf{R}^{n} \rightarrow \mathbf{C}$ is said to be a $(p, \infty)$ atom if

(i) $\operatorname{supp}(a) \subset B=B\left(x_{0}, r\right)$ for some ball $B\left(x_{0}, r\right):=\left\{x \in \mathbf{R}^{n}:\left|x-x_{0}\right| \leq\right.$ $r\}$,

(ii) $\|a\|_{\infty} \leq|B|^{-1 / p}$, and

(iii) $\int x^{\beta} a(x) d x=0$ for all multi-indices $\beta \in \mathbf{Z}_{+}^{n}$ with $0 \leq|\beta| \leq N:=$ $[n(1 / p-1)]$.

To prove that a function $m \in L^{\infty}\left(\mathbf{R}^{n}\right)$ belongs to $\mathscr{M}\left(H^{p}\right)$ it is sufficient to show that there exists a constant $C>0$ so that for every $(p, \infty)$ atom $a$ we have $\left\|(m \hat{a})^{\vee}\right\|_{H^{p}} \leq C$. Moreover, we only need to consider $(p, \infty)$ atoms with support in a ball centered at $x_{0}=0$. Furthermore, if $a$ is such an atom with 
$\operatorname{supp}(a) \subset B_{0}=B\left(0, r_{0}\right)$ for some $r_{0}>0$ and $\|a\|_{\infty} \leq\left|B_{0}\right|^{-1 / p}$ we can find a $k_{0} \in \mathbf{Z}$ so that $2^{k_{0}} \leq r_{0}<2^{k_{0}+1}$. Then $\operatorname{supp}(a) \subset B_{1}=B\left(0,2^{k_{0}+1}\right)$ and $\|a\|_{\infty} \leq 2^{n / p}\left|B_{1}\right|^{-1 / p}$. Thus $a$ can always be considered as a fixed multiple of an atom $\tilde{a}$ with support in a ball of radius $2^{k}$ for some $k \in \mathbf{Z}$ and $\|\tilde{a}\|_{\infty} \leq$ $\left|B\left(0,2^{k}\right)\right|^{-1 / p}$.

Hence, it follows from the nature of the assumptions we will make in Theorem 2.2 that we only need to consider a so-called unit atom $a$, that is

(i) $\operatorname{supp}(a) \subset B(0,1)$,

(ii) $\|a\|_{\infty} \leq 1$, and

(iii) $\int x^{\beta} a(x) d x=0$ for $0 \leq|\beta| \leq N$.

For $m \in L^{\infty}\left(\mathbf{R}^{n}\right)$ and $\delta>0$ define $\eta$ and $m_{\delta}$ as in the Introduction. Following [1] we next introduce a function $\psi \in C^{\infty}\left(\mathbf{R}^{n}\right)$ such that $\operatorname{supp}(\psi) \subset$ $\bar{A}_{0}, 0 \leq \psi(\xi) \leq 1$ and $\sum_{j=-\infty}^{\infty} \psi\left(2^{j} \xi\right)=1$ for all $\xi \neq 0$. Then, see [1, p. 44], for a unit atom $a$ we have

$$
m(\xi) \hat{a}(\xi)=\sum_{j=-\infty}^{\infty} m_{2^{j}}\left(2^{-j} \xi\right) \hat{a}_{2^{j}}\left(2^{-j} \xi\right),
$$

where $\hat{a}_{\delta}(\xi)=\hat{a}(\delta \xi) \psi(\xi)$ for $\delta>0$. Thus if $f:=(m \hat{a})^{\vee}$ we have

$$
f(x)=\sum_{j=-\infty}^{\infty} 2^{n j}\left(f_{j} * b_{j}\right)\left(2^{j} x\right)
$$

where $f_{j}(x)=\left(m_{2^{j}}\right)^{\vee}(x)$ and $b_{j}(x)=\left(\hat{a}_{2^{j}}\right)^{\vee}(x)$.

For ease of reference we state here some inequalities for the functions $b_{j}(x)$ that were proved in Lemma 1 of $\S 6$ in [1].

Let $r>0$ be given. Then

$$
\begin{array}{ll}
\left|b_{j}(x)\right| \leq C 2^{j(N+1)}(1+|x|)^{-r} & \text { if } j \leq 0, \\
\left|b_{j}(x)\right| \leq C 2^{-j n} & \text { if } j \geq 0, \\
\left|b_{j}(x)\right| \leq C 2^{-j n}|x|^{-r} & \text { if } j \geq 0 \text { and }|x|>2^{j+1} .
\end{array}
$$

Using these inequalities we can establish the following lemma.

Lemma 2.1. Let $0<p \leq 1$. Then

$$
\sum_{j=-\infty}^{\infty}\left(2^{n j(1-1 / p)}\left\|b_{j}\right\|_{p}\right)^{2}<\infty
$$

Proof. It follows from (2.1) that for $j \leq 0$,

$$
\left\|b_{j}\right\|_{p}^{p} \leq C 2^{j(N+1) p} \int(1+|x|)^{-r p} d x \leq C 2^{j(N+1) p}
$$

provided we choose $r$ sufficiently large. Thus

$$
\sum_{j=-\infty}^{0}\left(2^{n j(1-1 / p)}\left\|b_{j}\right\|_{p}\right)^{2} \leq C \sum_{j=-\infty}^{0}\left(2^{n j(1-1 / p)} 2^{j(N+1)}\right)^{2}<\infty,
$$

because $n(1-1 / p)+[n(1 / p-1)]+1>0$. Next, for $j \geq 1$ we have

$$
\begin{aligned}
\left\|b_{j}\right\|_{p}^{2} & \leq C\left(\left(\int_{|x| \leq 2^{j+1}}\left|b_{j}(x)\right|^{p} d x\right)^{2 / p}+\left(\int_{|x|>2^{j+1}}\left|b_{j}(x)\right|^{p} d x\right)^{2 / p}\right) \\
& :=C\left(P_{j}+Q_{j}\right) .
\end{aligned}
$$


To estimate $P_{j}$ we first apply Hölder's inequality.

$$
P_{j} \leq \int\left|b_{j}(x)\right|^{2} d x\left|B\left(0,2^{j+1}\right)\right|^{2 / p-1} .
$$

Substituting the definition of $\left(b_{j}\right)^{\vee}$ and a change of variables yields

$$
\begin{aligned}
P_{j} & \leq C 2^{n j(2 / p-1)} \int\left|\hat{a}\left(2^{j} \xi\right)\right|^{2}|\psi(\xi)|^{2} d \xi \\
& =C 2^{n j(2 / p-2)} \int|\hat{a}(\eta)|^{2}\left|\psi\left(2^{-j} \eta\right)\right|^{2} d \eta \leq C 2^{n j(2 / p-2)} \int_{\bar{A}_{j}}|\hat{a}(\eta)|^{2} d \eta .
\end{aligned}
$$

Therefore,

$$
\sum_{j=1}^{\infty} 2^{2 n j(1-1 / p)} P_{j} \leq C \sum_{j=1}^{\infty} \int_{\bar{A}_{j}}|\hat{a}(\eta)|^{2} d \eta \leq C\|a\|_{2}^{2} \leq C,
$$

because $a$ is a unit atom. To estimate $Q_{j}$ we use (2.3).

$$
Q_{j} \leq C\left(\int_{|x|>2^{j+1}}\left(2^{-n j}|x|^{-r}\right)^{p} d x\right)^{2 / p} \leq C\left(2^{-n j p} 2^{j(-r p+n)}\right)^{2 / p},
$$

provided we choose $r>n / p$. Therefore,

$$
\sum_{j=1}^{\infty} 2^{2 n j(1-1 / p)} Q_{j} \leq C \sum_{j=1}^{\infty} 2^{2 n j(1-1 / p-1+1 / p-r / n)}<C,
$$

because $r>0$. This completes the proof of the lemma.

In the proof of our main theorem we repeatedly use an inequality due to Peetre (see [5, Chapter 11] or [6, §1.5.3]). We state this inequality here for ease of reference later on.

Let $0<p \leq 1$ and let $\Omega$ be a compact subset of $\mathbf{R}^{n}$. Let $f, g \in L_{\Omega}^{p}:=$ $\left\{f \in \mathscr{S}^{\prime} \cap L^{p}: \operatorname{supp}(\hat{f}) \subset \Omega\right\}$. Then $f * g$ is well defined and

$$
\|f * g\|_{p} \leq C\|f\|_{p}\|g\|_{p} .
$$

Theorem 2.2. Let $0<p \leq 1$. Let $m \in L^{\infty}\left(\mathbf{R}^{n}\right)$ and assume

$$
\left(\left(m_{2^{j}}\right)^{\sim}\right)_{j=-\infty}^{\infty} \in l^{2 p /(2-p)}\left(L^{p}\right) .
$$

Then $m \in \mathscr{M}\left(H^{p}\right)$.

Proof. Let $a$ be a unit atom. If $f=(m \hat{a})^{\vee}$ then

$$
f(x)=\sum_{j=-\infty}^{\infty} 2^{n j}\left(f_{j} * b_{j}\right)\left(2^{j} x\right) .
$$

Thus, for $0<p \leq 1$ we have

$\|f\|_{p}^{p} \leq C \int \sum_{j=-\infty}^{\infty} 2^{n j p}\left|\left(f_{j} * b_{j}\right)\left(2^{j} x\right)\right|^{p} d x \leq C \sum_{j=-\infty}^{\infty} 2^{n j(p-1)} \int\left|\left(f_{j} * b_{j}\right)(u)\right|^{p} d u$.

Now we observe that $f_{j}(x)=\left(m_{2^{j}}\right)^{\vee}(x)$, so that $\left(f_{j}\right)^{\wedge}(\xi)=m\left(2^{j} \xi\right) \eta(\xi)$ and, hence, $\operatorname{supp}\left(f_{j}\right)^{\wedge} \subset\{|\xi| \leq 4\}$. Similarly, $b_{j}(x)=\left(\hat{a}_{2^{j}}\right)^{\vee}(x)$ so that $\left(b_{j}\right)^{\wedge}(\xi)=$ 
$\hat{a}\left(2^{j} \xi\right) \psi(\xi)$ and, hence, $\operatorname{supp}\left(b_{j}\right)^{\wedge} \subset\{|\xi| \leq 2\} \subset\{|\xi| \leq 4\}$. Thus, according to Peetre's inequality (2.4), we have for $0<p \leq 1$,

$$
\begin{aligned}
\|f\|_{p}^{p} & \leq C \sum_{j=-\infty}^{\infty} 2^{n j(p-1)}\left\|f_{j}\right\|_{p}^{p}\left\|b_{j}\right\|_{p}^{p} \\
& \leq C\left(\sum_{j=-\infty}^{\infty}\left(2^{n j(p-1)}\left\|b_{j}\right\|_{p}^{p}\right)^{2 / p}\right)^{p / 2}\left(\sum_{j=-\infty}^{\infty}\left(\left\|f_{j}\right\|_{p}^{p}\right)^{2 /(2-p)}\right)^{(2-p) / 2} \\
& \leq C\left(\sum_{j=-\infty}^{\infty}\left(2^{n j(1-1 / p)}\left\|b_{j}\right\|_{p}\right)^{2}\right)^{p / 2}\left(\sum_{j=-\infty}^{\infty}\left\|\left(m_{2^{j}}\right)^{\wedge}\right\|_{p}^{2 p /(2-p)}\right)^{(2-p) / 2} \leq C,
\end{aligned}
$$

by Lemma 2.1 and the assumption of the theorem. Thus $m \in \mathscr{M}\left(H^{p}, L^{p}\right)$. For $p=1$ this immediately implies that $m \in \mathscr{M}\left(H^{1}\right)$. In case $0<p<1$ we need to apply an argument as on p. 48 of [1]. Thus, for $\varepsilon>0$ and $\beta$ a multi-index in $\mathbf{Z}_{+}^{n}$, let $R$ be determined by

$$
\widehat{R}(\xi)=\xi^{\beta} e^{-\varepsilon|\xi|} /|\xi|^{|\beta|} .
$$

For $f=(m \hat{a})^{\vee}$ we have $R * f=(\widehat{R} m \hat{a})^{\vee}=(\bar{m} \hat{a})^{\vee}$ where $\bar{m}=\widehat{R} m$. We shall show that $\bar{m}$ again satisfies the assumptions of the theorem. Let $\phi \in C^{\infty}\left(\mathbf{R}^{n}\right)$ with $\operatorname{supp}(\phi) \subset\{1 / 8 \leq|\xi| \leq 8\}$ and $\phi(\xi)=1$ on $\{1 / 4 \leq|\xi| \leq 4\}$, so that $\eta \phi=\eta$. For every $j \in \mathbf{Z}$ we have

$$
\bar{m}_{2^{j}}(\xi)=\widehat{R}\left(2^{j} \xi\right) \phi(\xi) m\left(2^{j} \xi\right) \eta(\xi):=\widehat{Q}_{2^{j}}(\xi) m_{2^{j}}(\xi)
$$

so that $\left(\bar{m}_{2^{j}}\right)^{\vee}(x)=Q_{2^{j}} *\left(m_{2^{j}}\right)^{\vee}(x)$. Applying Peetre's inequality we obtain

$$
\left\|\left(\bar{m}_{2^{j}}\right)^{\wedge}\right\|_{p} \leq C\left\|Q_{2^{j}}\right\|_{p}\left\|\left(m_{2^{j}}\right)^{\wedge}\right\|_{p} \leq C\left\|\left(m_{2^{j}}\right)^{\wedge}\right\|_{p},
$$

according to an inequality proved on p. 49 of [1]. Consequently, $\|R * f\|_{p} \leq C_{\beta}$ and from this we may conclude that $m \in \mathscr{M}\left(H^{p}\right)$ in case $0<p<1$. This completes the proof of Theorem 2.2.

Remark. As mentioned earlier, Theorem 2.2 is the direct analogue on $\mathbf{R}^{n}$ of the multiplier theorem for Hardy spaces on Vilenkin groups given in Corollary 2.3 of [3]. To state this corollary explicitly we first need to introduce some notation. A locally compact Vilenkin group is a locally compact abelian topological group $G$ containing a strictly decreasing sequence of compact open subgroups $\left(G_{n}\right)_{-\infty}^{\infty}$ so that $\bigcup_{-\infty}^{\infty} G_{n}=G, \bigcap_{-\infty}^{\infty} G_{n}=\{0\}$, and $\sup \left\{\operatorname{order}\left(G_{n} / G_{n+1}\right): n \in \mathbf{Z}\right\}<\infty$. We denote its dual group by $\Gamma$ and we set $\Gamma_{n}=\{\gamma \in \Gamma: \gamma(x)=1$ for all $\left.x \in G_{n}\right\}$. We choose Haar measures $\mu$ on $G$ and $\lambda$ on $\Gamma$ so that $\mu\left(G_{0}\right)=$ $\lambda\left(\Gamma_{0}\right)=1$. Furthermore, for $\phi \in L^{\infty}(\Gamma)$ and $j \in \mathbf{Z}$ we set $\phi^{j}=\phi \chi_{\Gamma_{j+1} \backslash \Gamma_{j}}$ (see [3] for further details). In [3] the following result was obtained.

Theorem 0 \& Q. Let $0<p \leq 1$ and let $\phi \in L^{\infty}(\Gamma)$. If

$$
\sum_{j=-\infty}^{\infty}\left(\left(\lambda\left(\Gamma_{j}\right)\right)^{1 / p-1}\left\|\left(\phi^{j}\right)^{\vee}\right\|_{p}\right)^{2 p /(2-p)}<\infty,
$$

then $\phi \in \mathscr{M}\left(H^{p}(G)\right)$.

To further elucidate the similarity between Theorem 2.2 and Theorem $\mathrm{O} \&$ $\mathrm{Q}$, observe that the functions $\phi^{j}$ are comparable with the restrictions $\tilde{m}_{2^{j}}$ of 
$m$ defined by $\tilde{m}_{2^{j}}(\xi)=m(\xi) \eta\left(2^{j} \xi\right)$, rather than the functions $m_{2^{j}}$ as defined by Baernstein and Sawyer. It requires only an easy computation to show that

$$
\left(m_{2^{j}}\right)^{\wedge}(x)=2^{-j n}\left(\tilde{m}_{2^{j}}\right)^{\wedge}\left(2^{-j} x\right)
$$

so that the assumption (2.5) in Theorem 2.2 is equivalent to

$$
\sum_{j=-\infty}^{\infty}\left(2^{n j(1 / p-1)}\left\|\left(\tilde{m}_{2^{j}}\right)^{-}\right\|_{p}\right)^{2 p /(2-p)}<\infty
$$

and, clearly, (2.7) is similar to (2.6).

\section{A SHARPNESS RESULT}

In Theorem 2.3 of [3] we proved the sharpness of Theorem $O \& Q$. In a similar vein we shall prove the sharpness of Theorem 2.2. As will be clear soon, the construction of the examples used in the proof of Theorem 3.1 was strongly influenced by the example given by Baernstein and Sawyer in their proof of Theorem $5 \mathrm{~b}$ in [1].

Theorem 3.1. Let $0<p \leq 1$ and let $q>2 p /(2-p)$. Then there exists an $m \in L^{\infty}\left(\mathbf{R}^{n}\right)$ such that

(a) $\left(\left(m_{2^{j}}\right) \wedge\right) \in l^{q}\left(L^{p}\right)$, and

(b) $m \notin \mathscr{M}\left(H^{p}\right)$.

Proof. We first consider the case $0<p<1$. Choose sequences $\left(\lambda_{i}\right)_{1}^{\infty}$ and $\left(w_{i}\right)_{1}^{\infty}$ so that

(i) $\sum\left(w_{i}\right)^{-q}<\infty$,

(ii) $0 \leq \lambda_{i} / w_{i}<1$ for all $i \in \mathbf{N}$,

(iii) $\sum\left(\lambda_{i}\right)^{2}<\infty$,

(iv) $\sum \lambda_{i} / w_{i}<\infty$, and

(v) $\sum\left(\lambda_{i} / w_{i}\right)^{p}=\infty$.

For example, if we first choose $\rho$ so that $\max \{1 / q, 1 / 2\}<\rho<1 / p-1 / 2$ and then $\sigma$ so that $1 / 2<\sigma<1 / p-\rho$ we may take $\lambda_{i}=i^{-\sigma}$ and $w_{i}=i^{\rho}$. Next, choose a sequence of integers $\left(k_{i}\right)_{1}^{\infty}$ so that $k_{1} \geq 10, k_{i+1}-k_{i} \geq 10$ for all $i \geq 1$ and $\lim _{i \rightarrow \infty}\left(k_{i+1}-k_{i}\right)=\infty$. Furthermore, let $\psi \in C^{\infty}\left(\mathbf{R}^{n}\right)$ so that $\operatorname{supp}(\psi) \subset\{|\xi| \leq 1\}$ and $\psi(\xi)=1$ on $\{|\xi| \leq 1 / 2\}$ and let $f_{0}(x)=\psi^{\vee}(x)$. Define $m: \mathbf{R}^{n} \rightarrow \mathbf{C}$ by

$$
m(\xi)=\sum_{j=1}^{\infty} \frac{1}{w_{j}} \psi\left(2^{-k_{j}}\left(\xi-2^{k_{j}+2} e_{1}\right)\right) \exp \left\{2 \pi i\left(2^{k_{j}}-2^{-k_{j}}\right) e_{1} \cdot\left(\xi-2^{k_{j}+2} e_{1}\right)\right\},
$$

where $e_{1}=(1,0, \ldots, 0) \in \mathbf{R}^{n}$. Then $m_{2^{l}}(\xi) \not \equiv 0$ only if we have $k_{j} \leq l \leq$ $k_{j}+5$ for some $k_{j}$. For such values of $l$ we have (we present here the details of the calculations for the case $l=k_{j}$ ):

$$
\left(m_{2^{k_{j}}}\right)^{\vee}(x)=\frac{1}{w_{j}} f_{0}\left(x+\left(2^{2 k_{j}}-1\right) e_{1}\right) e^{2 \pi i x \cdot 4 e_{1}} * \check{\eta}(x),
$$

so that Peetre's inequality implies that

$$
\mid\left(m_{2^{k_{j}}}\right)^{\wedge}\left\|_{p}^{p} \leq C\left(w_{j}\right)^{-p}\right\| f_{0}\left(x+\left(2^{2 k_{j}}-1\right) e_{1}\right)\left\|_{p}^{p}\right\| \check{\eta} \|_{p}^{p}=C\left(w_{j}\right)^{-p} .
$$

Thus,

$$
\sum_{i=-\infty}^{\infty}\left\|\left(m_{2^{i}}\right)^{\wedge}\right\|_{p}^{q} \leq C \sum_{j=1}^{\infty}\left(w_{j}\right)^{-q}<\infty
$$


by condition (i); that is, $m$ satisfies (a). Next we define the function $g: \mathbf{R}^{n} \rightarrow \mathbf{C}$ by

$$
g(\xi)=\sum_{j=1}^{\infty} \lambda_{j} \psi\left(\xi-2^{k_{j}+2} e_{1}\right) \exp \left\{2 \pi i 2^{-k_{j}} e_{1} \cdot\left(\xi-2^{k_{j}+2} e_{1}\right)\right\}:=\sum_{j=1}^{\infty} \lambda_{j} g_{j}(\xi) .
$$

We shall prove that $\check{g} \in H^{p}$ by showing that $\check{g}$ is a $(p, 2, b)$ molecule, see [2] for the definition of such molecules. Since $g(\xi)=0$ in a neighborhood of $\xi=0$, we have $\int x^{\beta} \check{g}(x) d x=0$ for all multi-indices $\beta$ with $|\beta| \geq 0$. Also, condition (iii) implies that

$$
\|\check{g}\|_{2}^{2}=\|g\|_{2}^{2} \leq \sum_{j=1}^{\infty}\left|\lambda_{j}\right|^{2}\|\psi\|_{2}^{2}<\infty .
$$

Furthermore, for every $j \in \mathbf{N}$,

$$
\left(g_{j}\right)^{\vee}(x)=f_{0}\left(x+2^{-k_{j}} e_{1}\right) \exp \left(2 \pi i 2^{k_{j}+2} x \cdot e_{1}\right) .
$$

Therefore, for any $r>0$ there exists $C>0$ so that

$$
\left|\left(g_{j}\right)^{\vee}(x)\right| \leq C\left(1+\left|x+2^{-k_{j}} e_{1}\right|\right)^{-r} .
$$

Let $b=[1 / p-1 / 2]+1$. Then, with

$$
\begin{gathered}
\Delta_{\xi}=-\sum_{i=1}^{n} \frac{\partial^{2}}{\partial \xi_{i}^{2}} \\
\left\||x|^{n b} \check{g}(x)\right\|_{2}^{2}=C\left\|\left(-\Delta_{\xi}\right)^{n b / 2} g\right\|_{2}^{2}=C\left\|\sum_{j=1}^{\infty} \lambda_{j}\left(-\Delta_{\xi}\right)^{n b / 2} g_{j}(\xi)\right\|_{2}^{2} \\
=C \sum_{j=1}^{\infty}\left|\lambda_{j}\right|^{2}\left\|\left(-\Delta_{\xi}\right)^{n b / 2} g_{j}(\xi)\right\|_{2}^{2},
\end{gathered}
$$

because $\operatorname{supp}\left(g_{i}\right) \cap \operatorname{supp}\left(g_{j}\right)=\varnothing$ whenever $i \neq j$. Consequently,

$$
\left\||x|^{n b} \check{g}(x)\right\|_{2}^{2} \leq C \sum_{j=1}^{\infty}\left|\lambda_{j}\right|^{2}\left\||x|^{n b}\left(g_{j}\right)^{\vee}(x)\right\|_{2}^{2} .
$$

Next, for every $j \in \mathbf{N}$ we have

$$
\begin{aligned}
\left\||x|^{n b}\left(g_{j}\right)^{\vee}(x)\right\|_{2}^{2} & \leq C \int|x|^{2 n b}\left(1+\left|x+2^{-k_{j}} e_{1}\right|\right)^{-2 r} d x \\
& \leq C\left(\int_{|x| \leq 1} \cdots+\int_{|x|>1} \cdots\right) .
\end{aligned}
$$

Clearly,

$$
\int_{|x| \leq 1}|x|^{2 n b}\left(1+\left|x+2^{-k_{j}} e_{1}\right|\right)^{-2 r} d x \leq C .
$$

Moreover, because $\left|x+2^{-k_{j}} e_{1}\right| \geq|x| / 2$ for $|x| \geq 1$, we also have

$$
\int_{|x|>1}|x|^{2 n b}\left(1+\left|x+2^{-k_{j}} e_{1}\right|\right)^{-2 r} d x \leq C
$$


for $r$ sufficiently large. Hence,

$$
\left\||x|^{n b} \check{g}(x)\right\|_{2}^{2} \leq C \sum_{j=1}^{\infty}\left|\lambda_{j}\right|^{2}<\infty .
$$

Thus, we may conclude that $\check{g} \in H^{p}$. Finally, we consider $m(\xi) g(\xi)$. It follows immediately from the definitions of $m(\xi)$ and $g(\xi)$ that

$$
m(\xi) g(\xi)=\sum_{j=1}^{\infty} \frac{\lambda_{j}}{w_{j}} \psi\left(\xi-2^{k_{j}+2} e_{1}\right) \exp \left(2 \pi i 2^{k_{j}} e_{1} \cdot \xi\right)
$$

so that

$$
(m g)^{\vee}(x)=C \sum_{j=1}^{\infty} \frac{\lambda_{j}}{w_{j}} f_{0}\left(x+2^{k_{j}} e_{1}\right) \exp \left(2 \pi i 2^{k_{j}+2} x \cdot e_{1}\right) .
$$

We will prove that $(m g)^{\vee} \notin H^{p}$. Obviously,

$$
\left\|(m g)^{\vee}\right\|_{1} \leq C \sum_{j=1}^{\infty}\left|\frac{\lambda_{j}}{w_{j}}\right|<\infty
$$

by condition (iv). To simplify the notation in what follows, let $B_{j}:=\{x \in$ $\left.\mathbf{R}^{n}:\left|x-2^{k_{j}} e_{1}\right| \leq 1\right\}$. Fix $j \in \mathbf{N}$; we have

$$
\begin{gathered}
\int_{B_{j}}\left|\frac{\lambda_{j}}{w_{j}} f_{0}\left(x+2^{k_{j}} e_{1}\right) \exp \left(2 \pi i 2^{k_{j}+2} x_{1}\right)\right|^{p} d x \\
=\left|\frac{\lambda_{j}}{w_{j}}\right|^{p} \int_{0 \leq|x| \leq 1}\left|f_{0}(u)\right|^{p} d u=C\left|\frac{\lambda_{j}}{w_{j}}\right|^{p} .
\end{gathered}
$$

Next, for $l<j$ we have

$$
\int_{B_{j}}\left|\frac{\lambda_{l}}{w_{l}}\right|^{p}\left|f_{0}\left(x+2^{k_{l}} e_{1}\right)\right|^{p} d x \leq\left|\frac{\lambda_{l}}{w_{l}}\right|^{p} \int_{B_{j}}\left(1+\left|x+2^{k_{l}} e_{1}\right|\right)^{-r p} d x \leq C 2^{-r p k_{j}},
$$

so that

$$
P_{j}:=\sum_{l=1}^{j-1} \int_{B_{j}}\left|\frac{\lambda_{l}}{w_{l}}\right|^{p}\left|f_{0}\left(x+2^{k_{l}} e_{1}\right)\right|^{p} d x \leq C j 2^{-r p k_{j}} \leq C 2^{-r p k_{j} / 2}
$$

Thus we see that $P_{j} \rightarrow 0$ as $j \rightarrow \infty$. For $l>j$ we have

$$
\begin{aligned}
\int_{B_{j}}\left|\frac{\lambda_{l}}{w_{l}}\right|^{p}\left|f_{0}\left(x+2^{k_{l}} e_{1}\right)\right|^{p} d x \\
\quad \leq C\left|\frac{\lambda_{l}}{w_{l}}\right|^{p} \int_{B_{j}}\left(1+\left|x+2^{k_{l}} e_{1}\right|\right)^{-r p} d x \leq C 2^{-r p k_{l}},
\end{aligned}
$$

so that

$$
Q_{j}:=\sum_{l=j+1}^{\infty} \int_{B_{j}}\left|\frac{\lambda_{l}}{w_{l}}\right|^{p}\left|f_{0}\left(x+2^{k_{l}} e_{1}\right)\right|^{p} d x \leq C \sum_{l=j+1}^{\infty} 2^{-r p k_{l}}
$$


and we see that $Q_{j} \rightarrow 0$ as $j \rightarrow \infty$. Therefore, for $j$ sufficiently large we have

$$
\int_{B_{j}}\left|(m g)^{\vee}(x)\right|^{p} d x \geq C\left|\frac{\lambda_{j}}{w_{j}}\right|^{p} \text {. }
$$

Hence $(m g)^{\vee} \notin L^{p}$ and we may conclude that $(m g)^{\vee} \notin H^{p}$. Thus $m \notin$ $\mathscr{M}\left(H^{p}\right)$, which concludes the proof of Theorem 2.3 for $0<p<1$.

In case $p=1$ choose $\rho$ and $\sigma$ so that $1 / q<\rho<1 / 2<\sigma<1-\rho$. Choose the sequence $\left(k_{j}\right)_{1}^{\infty}$ as before and let the function $\psi \in C^{\infty}\left(\mathbf{R}^{n}\right)$ satisfy $\operatorname{supp}(\psi) \subset\{|\xi| \leq 1\}$ and $\psi(\xi)=1$ on $\{|\xi| \leq 1 / 2\}$. Define $m: \mathbf{R}^{n} \rightarrow \mathbf{C}$ by

$$
m(\xi)=\sum_{j=1}^{\infty} j^{-\rho} \psi\left(2^{k_{j}}\left(\xi-2^{k_{j}} e_{1}\right)\right) \text {. }
$$

Then it is easy to see that

$$
\sum_{j=1}^{\infty}\left\|\left(m_{2^{j}}\right) \uparrow\right\|_{1}^{q} \leq C \sum_{i=1}^{\infty} i^{-\rho q}<\infty,
$$

that is, $m$ satisfies (a). Next, define $g: \mathbf{R}^{n} \rightarrow \mathbf{C}$ by

$$
g(\xi)=\sum_{j=1}^{\infty} j^{-\sigma} \psi\left(\xi-2^{k_{j}} e_{1}\right)
$$

Then $g^{\vee} \in H^{1}$, see p. 66 of [1], and

$$
f(x):=(m g)^{\vee}(x)=\sum_{j=1}^{\infty} j^{-\rho-\sigma} 2^{-n k_{j}} \psi^{\vee}\left(2^{-k_{j}} x\right) \exp \left(2 \pi i 2^{k_{j}} e_{1}\right) .
$$

A computation like in [1] shows that for $j$ sufficiently large we have

$$
\int_{A_{k_{j}}}|f(x)| d x \geq C j^{-\rho-\sigma} .
$$

Since $\rho+\sigma<1$ we may conclude that $f \notin L^{1}$. Thus $f \notin H^{1}$ and this implies that $m \notin \mathscr{M}\left(H^{1}\right)$. This completes the proof of Theorem 3.1.

Concluding remark. We have also considered multipliers on Lebesgue spaces $L^{p}(\mathbf{R})$ and $L^{p}(G)$, where $G$ is a locally compact Vilenkin group, and where the assumption on the multiplier is expressed in terms of a mixed-norm condition. The techniques for proving multiplier theorems for Lebesgue spaces are very different from the techniques used in this paper. The results we have obtained for such multipliers on $L^{p}(\mathbf{R})$ and on $L^{p}(G), 1<p<\infty$, are presented in [4].

\section{REFERENCES}

1. A. Baernstein II and E. T. Sawyer, Embedding and multiplier theorems for $H^{p}\left(\mathbf{R}^{n}\right), \operatorname{Mem}$. Amer. Math. Soc., No. 318, Amer. Math. Soc., Providence, RI, 1985.

2. J. Garcia-Cuerva and J. L. Rubio de Francia, Weighted norm inequalities and related topics, North-Holland Math. Studies, vol. 116, North-Holland, Amsterdam, 1985.

3. C. W. Onneweer and T. S. Quek, Multipliers for Hardy spaces on locally compact Vilenkin groups, J. Austral. Math. Soc. (to appear). 
4. $ـ$, On $L^{p}$-multipliers of mixed-norm type, J. Math. Anal. Appl. (to appear).

5. J. Peetre, New thoughts on Besov spaces, Duke Univ. Math. Ser., Duke Univ., Durham, NC, 1976.

6. H. Triebel, Theory of function spaces, Birkhäuser-Verlag, Basel, 1983.

Department of Mathematics, University of New Mexico, Albuquerque, New Mexico 87131

E-mail address: onneweer@gauss .unm.edu

Department of Mathematics, National University of Singapore, Singapore 0511, RePUBLIC OF SINGAPORE

E-mail address: matqts\%nusvm.bitnet@cunyvm. cuny.edu 